\title{
Early detection of anomalies in dam performance: a methodology based on boosted regression trees
}

\author{
F. Salazar ${ }^{1 *}$ M. Á. Toledo ${ }^{2}$ J. M. González ${ }^{1}$ and E. Oñate ${ }^{1}$ \\ ${ }^{1}$ International Center for Numerical Methods in Engineering (CIMNE). Campus Norte UPC. Gran Capitán s/n. 08034. \\ Barcelona, Spain \\ ${ }^{2}$ Technical University of Madrid (UPM). Department of Civil Engineering: Hydraulics, Energy and Environment. \\ Profesor Aranguren s/n, 28040, Madrid, Spain
}

\begin{abstract}
SUMMARY
The advances in information and communication technologies led to a general trend towards the availability of more detailed information on dam behaviour. This allows applying advanced data-based algorithms in its analysis, which has been reflected in an increasing interest in the field. However, most of the related literature is limited to the evaluation of model prediction accuracy, whereas the ulterior objective of data analysis is dam safety assessment. In this work, a machine learning algorithm (boosted regression trees) is the core of a methodology for early detection of anomalies. It also includes a criterion to determine whether certain discrepancy between predictions and observations is normal, a procedure to compute a realistic estimate of the model accuracy, and an original approach to identify extraordinary load combinations. The performance of causal and non-causal models is assessed in terms of their ability to detect different types of anomalies, which were artificially introduced on reference time series generated with a numerical model of a 100-m high arch dam. The final approach was implemented in an on-line application to visualise the results in an intuitive way to support decision making. Copyright (c) 2010 John Wiley \& Sons, Ltd.
\end{abstract}

Received ...

KEY WORDS: machine learning; dam safety; dam monitoring; boosted regression trees; anomaly detection

\section{INTRODUCTION}

Dam safety is an area of growing interest: our societies demand increasing safety levels, and the average age of dams is high in many countries, which increases the need for control and maintenance operations. The advances in information and communication technologies led to relevant improvements in the performance of monitoring systems, both in terms of accuracy and reliability of the devices, security of the communications and reading frequency. All this resulted in more information available on the behaviour of the structure [1].

\footnotetext{
${ }^{*}$ Correspondence to: International Center for Numerical Methods in Engineering (CIMNE). Campus Norte UPC. Gran Capitán s/n. 08034. Barcelona, Spain. E-mail: fsalazar@cimne.upc.edu

Copyright (c) 2010 John Wiley \& Sons, Ltd.
}

Prepared using stcauth.cls [Version: 2010/05/13 v2.00] 
This increase in the amount of available data led to the use of more powerful tools for its analysis, from enhanced versions of the multiple linear regression (e.g. [2]), up to algorithms developed in the field of machine learning, such as neural networks [3], support vector machines (SVM) [4], [5], or adaptive neuro-fuzzy inference systems (ANFIS) [6], among others [7], [8].

However, these methods are still not widely applied by practitioners, who mostly limit the data analysis to graphical exploration of the time series of data [9], along with simple statistical models [1], [10].

The vast majority of examples of application of advanced tools focus on the development of behaviour models to predict the value of a given response variable of the dam (e. g. radial displacement) as a function of the loads. The prediction is compared to the actually observed data and some error index is computed. In most cases, the results are more accurate than those obtained by conventional methods (e.g. [3]).

In general, these techniques offered some advantages over conventional statistical methods, in terms of greater accuracy, flexibility, or ability to interpret dam behaviour [11].

However, the main objective of dam safety is to prevent failures, for which anomalies need to be detected at early stage. The capability of predictive models to identify anomalies has been much less frequently studied. Mata et al. [12] developed a model based on linear discriminant analysis for the early detection of developing failure scenarios. This methodology belongs to the Type 2 among those defined by Hodge and Austin [13]: the system is trained with both normal and abnormal behaviour data, and classifies new inpyts as belonging to one of
of this approach is that the failure mode must be defined beforehar
accuracy to provide the training data. Herce, the system is specific
Jung et ab. [14] used a similar approach: abnormal situatio discrepancy between the model predictions and the observed data. This method focuses on embankment dam piezometer data and only the resservoir level is considered as external variable free at https/WwW.Scipedia.com to download the version without the
(although they acknowledge that the rainfall can also be influential). It is not clear whether this

methodology could be applied to other dam typologies or response variables.

Cheng and Zeng [15] presented a methodology based on the definition of some control limits, which depend on the prediction error of a regression model. In addition, they proposed a classification of anomalies based on the trend of the deviation and on how the overall deviance is distributed among the devices considered. It has the advantage of being simultaneously applied to a set of devices, although the case study presented is simple and the test period considered very short (30 days), as compared to the available data (1555 days).

Other examples of application of advanced tools together with prediction intervals have been published by Gamse and Oberguggenberger [16], who employed the procedure of probabilistic quality control, Yu et al. [10], based on principal component analysis (PCA), Kao and Loh [17], who used PCA together with neural networks (NN), Li et al. [18], who considered the autocorrelation of the residuals and Loh et al. [19], who presented models for short and long term prediction.

Most of these works follow a conceptually similar methodology: a prediction model is built, the density function of the residuals is calculated and used to define the prediction intervals, which are applied to detect anomalies. In all cases, the efficiency was verified by means of its application to a short period of records. As an exception, Jung et al. [14] and Mata et al. [12] used abnormal data obtained from finite element models (FEM). 
The main differences among authors lie in the prediction method used (parametric or nonparametric; static or autoregressive, etc.). In this article, a similar methodology is presented, with some innovative features:

- The prediction model is based on boosted regression trees (BRTs), which showed to be more accurate than other machine learning and statistical tools in previous works [8].

- Causal, non-causal and auto-regressive models are considered and jointly analysed.

- Artificially-generated data are taken as reference. They were obtained from a FEM model considering the coupling between thermal and hydrostatic loads. This allows to identify normal and abnormal behaviour, as observed by some authors ([14], [12]). In this work, the FEM results are compared to actually observed data to verify their reliability.

- A methodology is proposed to neglect false anomalies due to the occurrence of extraordinary loads. It is based on the values of the two main actions (thermal and hydrostatic).

- Three types of anomalies are considered, affecting both to isolated devices and to the whole structure.

- Although radial displacements in an arch dam were selected for the case study, the method can be applied to other dam typologies and response variables. Moreover, it adapts well to different amount and type of input variables, due to the great flexibility and robustness of
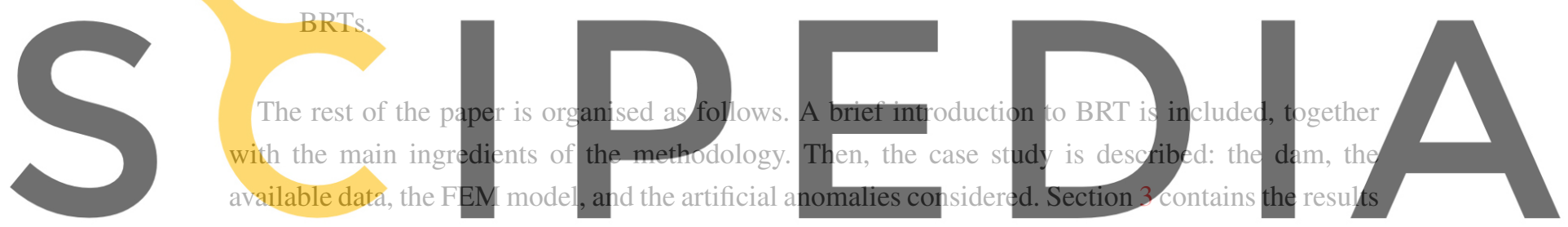

in terms of ability to detect different types of anomalies. The final version was implemented in an

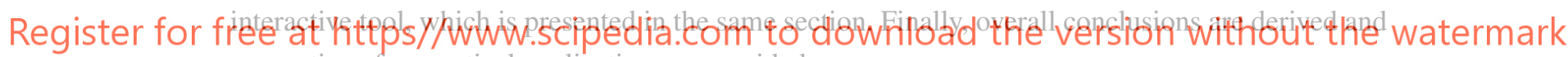
suggestions for practical application are provided.

\section{METHODS}

In previous studies, BRTs showed to be appropriate to build predictive models, mainly because of its high accuracy and flexibility [8]. The algorithm was further analysed in terms of model interpretation, and it was verified that useful information can be drawn as regards dam performance [11]. As a result, BRT was selected in this work as the predictive model, though the overall methodology may also be employed with other algorithms.

In what follows, $Y \in \mathbb{R}$ stands for the output variable (radial displacement), which is estimated as a function of some inputs $X$ (e.g. reservoir level, air temperature): $Y \approx \hat{Y}=F(X)$. The observed values are denoted as $\left(x_{i}, y_{i}\right), i=1, \ldots, N$, where $N$ is the number of observations. Each $x_{i}$ is a vector with $p$ components, each of which is referred to as $x_{i}^{j}$. Similarly, $X^{j}, j=1, \ldots, p$ stands for each dimension of the input space.

The outputs considered correspond to 8 radial displacement in 4 plumb lines ( 2 measurements per each plum line). The employed notation and their location within the dam body are shown in Figure 7. 


\subsection{Boosted regression trees}

BRT models belong to the category of ensemble methods, because the prediction is based on the contribution of a number of simple models (weak or base learners). In particular, two algorithms are combined in BRTs: decision trees [20] for the base learners, and boosting [21] for averaging. The fundamentals of both algorithms and that of BRTs can be found in the scientific literature (e.g. [22], [23], [24]), as well as in previous studies ([11]). For the sake of completeness, a brief introduction follows.

Regression trees are based on the recursive division of the training space into disjointed regions. The prediction is generally the mean of the output variable for the observations within each region. They were first proposed by Breiman et al. [20] and underwent high degree of development from then on.

In the general case, when several inputs are considered, the best split point for each is calculated, and that resulting in greater error reduction is chosen. This procedure allows for automatic selection of the most relevant predictors.

Regression trees are robust, require little data pre-processing, and can automatically reproduce non-linear relations, as well as interaction among predictors. By contrast, they are unstable, i. e., small variations in the training data may result in highly different results [24].

Boosting is a general procedure to build ensemble predictive tools [21], based on the combination
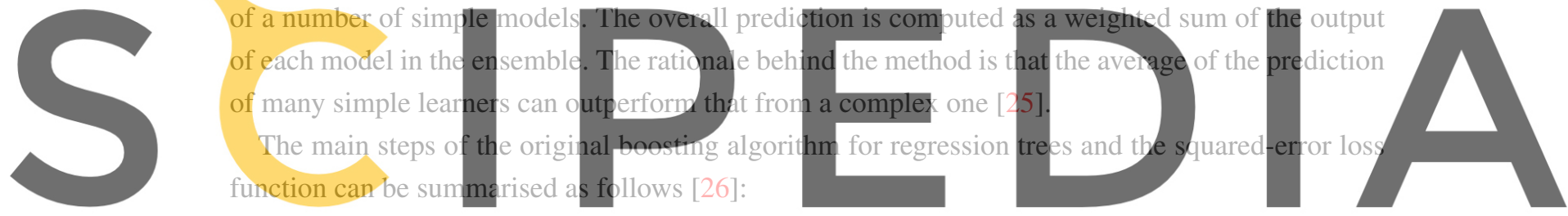

\section{Register for free at https//www.scipedia.com to download the version without the watermark}

1. Start predicting with the average of the observations (constant):

$$
F_{0}(X)=f_{0}(X)=\bar{y}_{i}
$$

2. For $m=1$ to $M$

(a) Compute the prediction error on the training set:

$$
\tilde{y}_{i}=y_{i}-F_{m-1}\left(x_{i}\right)
$$

(b) Draw a random sub-sample of the training set $\left(S_{m}\right)$

(c) Consider $S_{m}$ and fit a new regression tree to the residuals of the previous ensemble:

$$
\tilde{y}_{i} \approx f_{m}(X), i \in S_{m}
$$

(d) Update the ensemble:

$$
F_{m}(X) \Leftarrow F_{m-1}(X)+f_{m}(X)
$$

3. $F_{M}$ is the final model 
A regularisation parameter $\nu \in(0,1)$ is typically added to avoid over-fitting, so that step (d) turns into:

$$
F_{m}(X) \Leftarrow F_{m-1}(X)+\nu \cdot f_{m}(X)
$$

Based on the results of previous studies [8], [11], the models employed in this work initially contained 1,000 trees of two levels (four leaves) which were later pruned to the final shape via 5-fold cross-validation. The regularisation parameter $\nu$ was set to 0.01 . All the calculations were performed in the R environment [27] with the gbm library [28].

\subsection{Prediction intervals}

As mentioned above, most of the published works on the application of data-based models in dam monitoring are limited to the assessment of the model accuracy. However, the main practical utility of these models is the early detection of anomalies, for which it is necessary to compare the predictions with monitoring readings, and verify whether they fall within a predefined range. If the residual density function follows a normal distribution, that range can be defined in terms of the standard deviation of the residuals. For example, Kao and Loh [17] presented the 99\% prediction intervals for models based on neural networks, while Jung et al [14] tested 1, 2 and 3 standard deviations of the residuals as the width of the prediction interval.

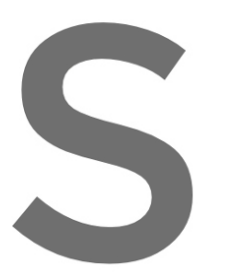

Based on previous studies with models based on BRTs [20], the prediction interval in this work was set to $\left[\mu-2 s d_{\text {res, }} \mu+2 s d_{\text {res }}\right.$, being $\mu$ and $s d_{\text {res }}$ the mean and the statard deviation of
the residuals, respectively. Special attention was paid to the determination of a realistic residual
distribution. It is well known that the acuracy of a machine learning prediction model must be
calculated from a data set not used for model fitting [30] (validation set). In the case of time series,
this validation set should be more recent in time than the training data, since in practice the model is used for predicting a time period subsequent to the training data [31].

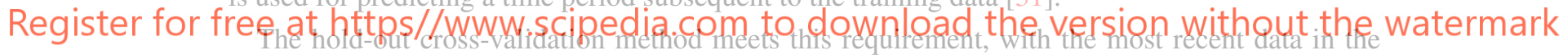
hold-out set (Figure 1).

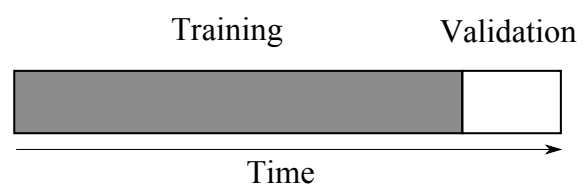

Figure 1. Hold-out cross-validation scheme.

However, this implies discarding the most recent data for the model fit, which are generally the most useful, since they represent the most similar behaviour to that to be predicted (assuming there may be a gradual change in behaviour over time). Moreover, the validation data may be biased, if they correspond, for instance, to a especially warm (or cold) period.

To overcome these drawbacks while maintaining good estimate of the prediction error, an approach based on the hold-out cross validation method suggested by Arlot and Celisse [31] for non-stationary time series data was employed.

The proposed method takes into account the following specific aspects of dam behaviour: a) changes in the dam-foundation system are generally gradual, and b) dam behaviour models are typically revised annually, coinciding with the update of safety reports. 
Let us consider that a behaviour model is to be fitted at the beginning of year $Z_{i}$, to be applied for anomaly detection during that year. The available data corresponds to the years $Z_{1} \ldots Z_{i-1}$, with $Z_{1}$ being the initial year of dam operation. With the simple hold-out method, a model is fitted with data in years $Z_{1} \ldots Z_{i-2}$, whose accuracy is evaluated on data in $Z_{i-1}$.

In this work, a minimum training period of 5 years was considered. This value was chosen in view of a) the results of previous studies [8], and b) the evolution of model accuracy on the reference data, as described in section 3.2. Then, an iterative process is followed to reduce potential bias in the loads during $Z_{i-1}$. A set of predictions is generated as follows:

- For $k=5 \ldots i-2$

- Fit a model $M_{k}$ trained with the period $Z_{1} \ldots Z_{k}$.

- Compute $R_{k}$ as the residuals of $M_{k}$ when predicting year $Z_{k+1}$.

- Compute the mean $\left(\mu_{k}\right)$ and standard deviation $\left(s d_{r e s, k}\right)$ of $R_{k}$

At the end of the process, residuals for a set of models $M_{k}, k=5 \cdots i-2$ are obtained, with the particularity that they are computed over different time periods, always subsequent to the training $\operatorname{set}\left(Z_{6} \cdots Z_{i}-1\right)$. That is, the amount of observations in the training sample increases, and is used to predict the following year. The potential bias of some abnormal loads for one year is compensated by averaging, while a realistic prediction error is achieved, since it is always based on precedent data.

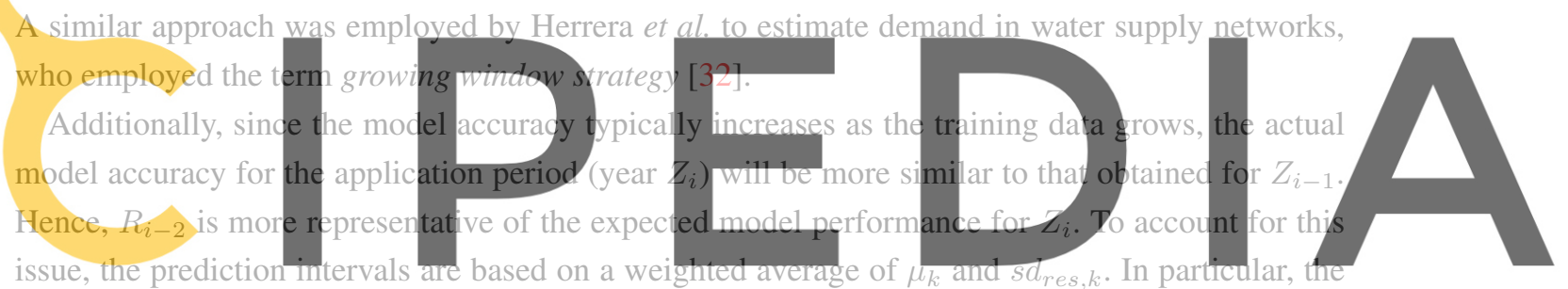
issue, the prediction intervals are based on a weighted average of $\mu_{k}$ and $s d_{r e s, k}$. In particular, the

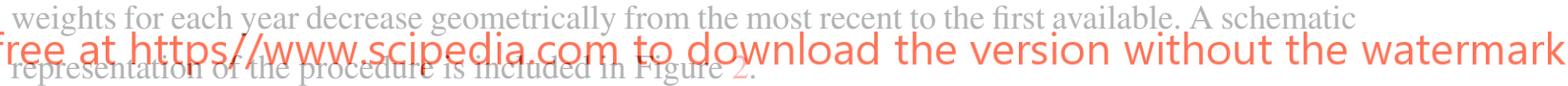

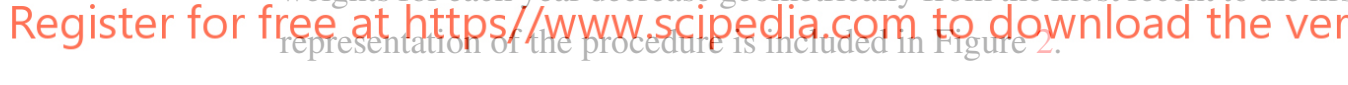

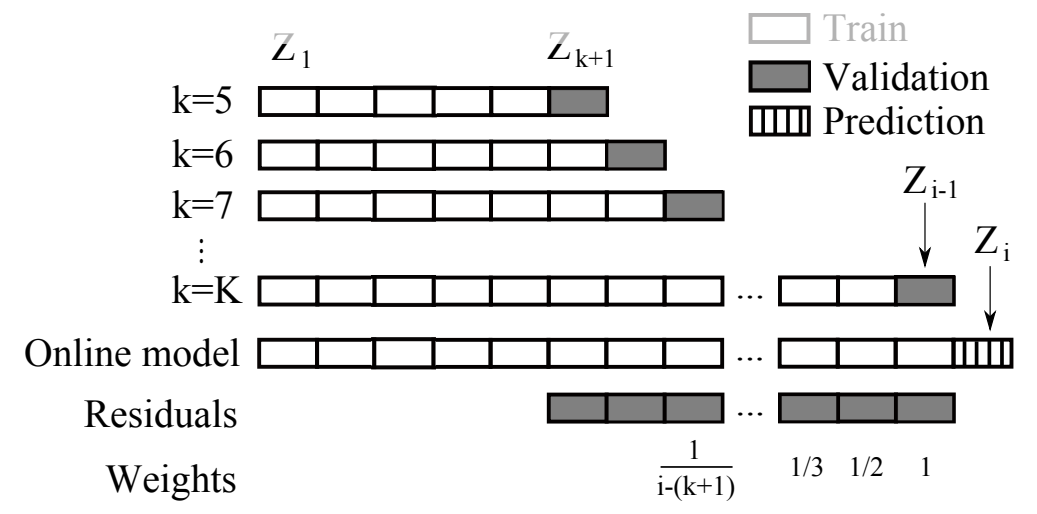

Figure 2. Graphical representation of the weighted growing-window cross-validation procedure. The prediction interval is estimated as a function of the weighted average of the standard deviation of the residuals for previous years, each one is computed from a model trained with a different training set.

Finally, to take advantage of all the available data, a model is fitted with the entire period $Z_{1} \ldots Z_{i-1}$, with which the predictions for the following year $\left(Z_{i}\right)$ are computed.

Since the test set becomes part of the validation period in the subsequent years, the residuals generated during the application of the model in the test period can be added to those computed for 
previous years, so that there is no need to repeat the whole process: the previous residuals can be employed to obtain the new prediction interval, after updating the correspondent weights.

\subsection{Causal and non-causal models}

BRT models are robust against the presence of uninformative or highly correlated predictors [21], [11]. Hence, variable selection is much less influential for tree-based methods than for other machine learning tools [33].

In the vast majority of published works, variables correspondent to environmental actions are considered as predictors: air temperature and hydrostatic load. Also, a time-dependent term is typically included, to identify possible variations in dam behaviour over the period of analysis. This criterion leads to causal models, since there is some causality relation between each input and the dam response, which can be identified by means of model interpretation [11]. This approach was also followed in this work to build the Causal models, though several variables derived from those actually measured at the dam site (reservoir level and the average daily temperature) were also included. They are listed in Table I. A priori, a model of this type is expected to detect reading errors and changes in dam behaviour. However, its accuracy might be improved, since the response of the dam may depend on variables not available, such as the maximum and minimum daily temperatures, or the solar radiation.
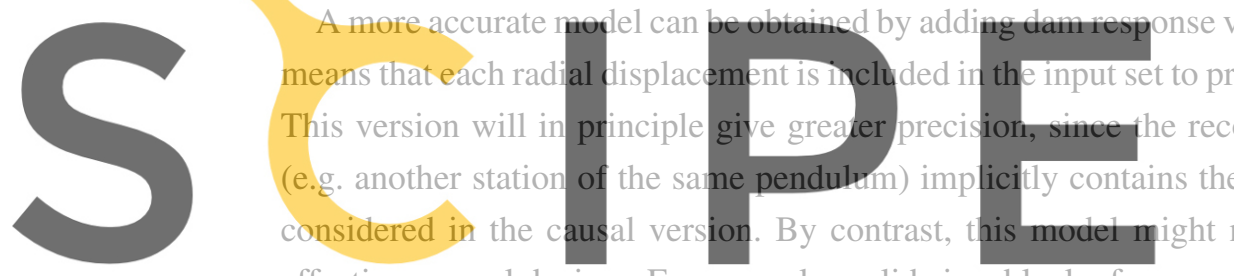

affecting several devices. For example, a slide in a block of a concrete gravity dam will be reflected

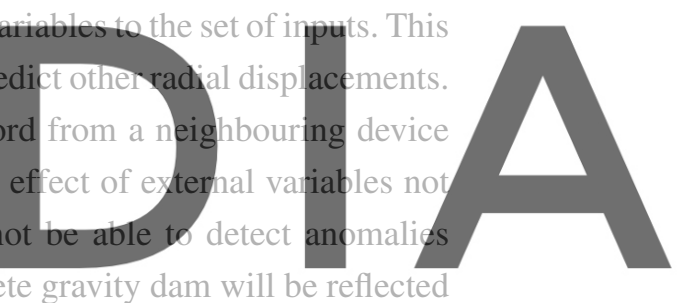

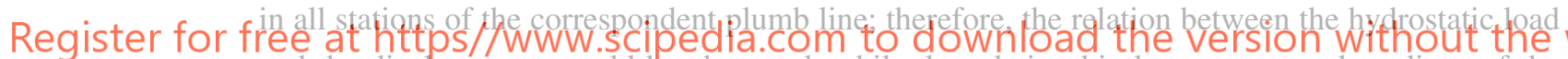
and the displacement would be abnormal, while the relationship between several readings of the

same pendulum could be normal. These models are termed Non-Causal herein.

A further degree of complexity can be incorporated by considering the lagged values of noncausal variables as predictors. This kind of models are frequently termed auto-regressive with exogenous inputs (ARX)*, and were previously employed in dam safety [34], [6]. Specifically, the response at time $t_{i}$ is estimated based on the readings at $t_{i-1}$ and $t_{i-2}$, both for the variable to predict and other response variables.

One of the objectives of this work is to test the ability of all three models to detect various types of abnormalities, and draw conclusions for practical purposes.

\subsection{Case study}

La Baells dam is a double-curvature arch dam located in the Llobregat river, in the Barcelona region (Spain). The crest length is $403 \mathrm{~m}$, whereas the maximum height above foundation is $102 \mathrm{~m}$. Monitoring data were provided by the Catalan Water Agency for the period 1981-2008. These data

\footnotetext{
*The ARX model is also non-causal, in the sense that variables with non-causal relation with the outputs are included as predictors. The acronym ARX was employed to distinguish both models when necessary, although they are occasionally jointly referred to as "non-causal models". For the sake of clarity, the capitalised version ("Non-Causal") is used to specifically refer to the second model, excluding the ARX.
} 
Table I. Predictor variables considered for the Causal BRT model.

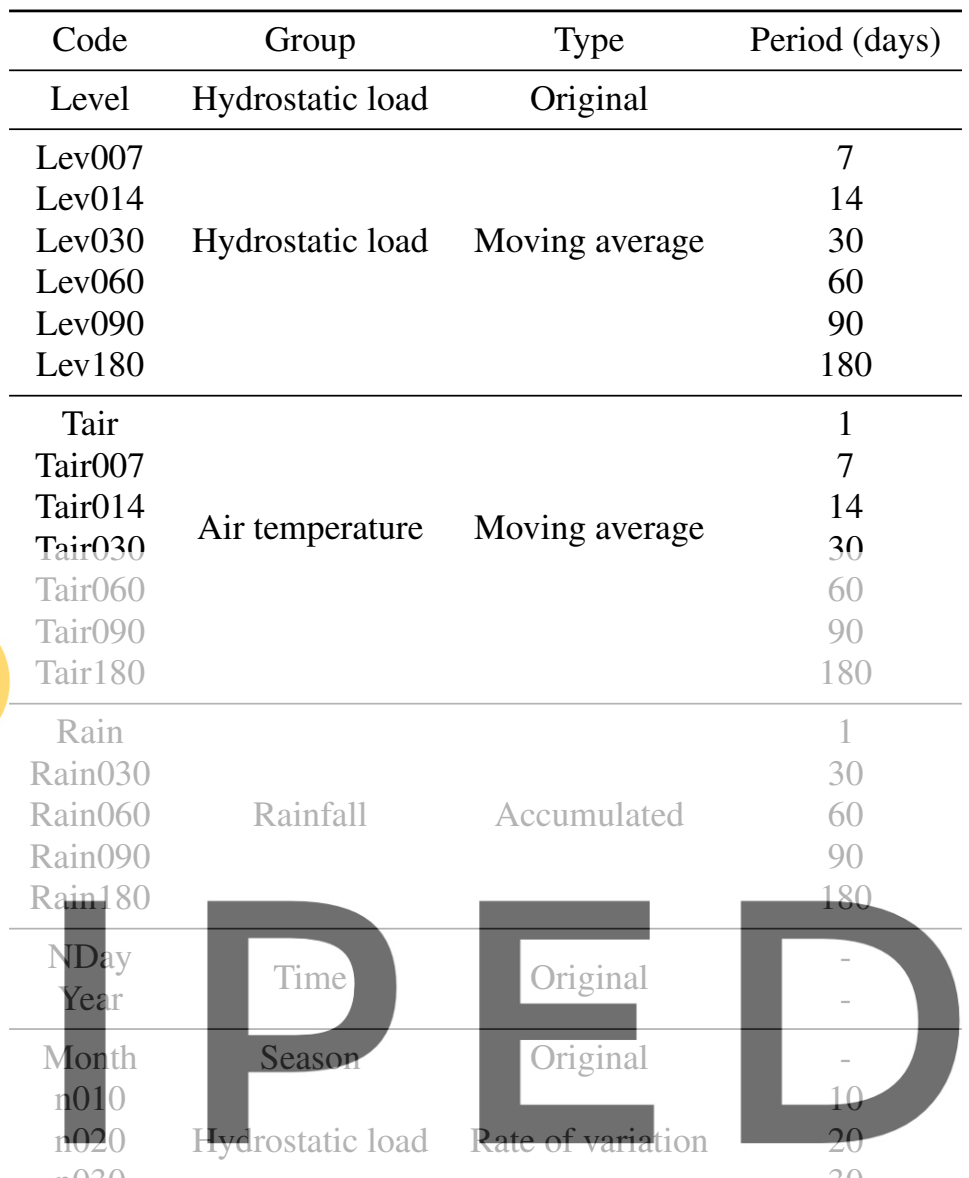

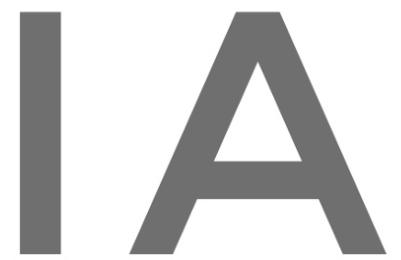

Register for free at https//www.scipedia.com to download the version without the watermark

correspond both to environmental and response variables. In this work, the air temperature (Figure

3) and the reservoir level (Figure 4) time series were considered as inputs to a finite element (FE) model. The results of this model in terms of radial displacements at the location of the pendulums were extracted and compared to the actual measurements (Figure 5). The objective was to check that the FE model could provide realistic data to generate reference time series of dam behaviour. These artificial data are free from any temporal variation (the reference numerical model does not vary with time; only environmental loads do).

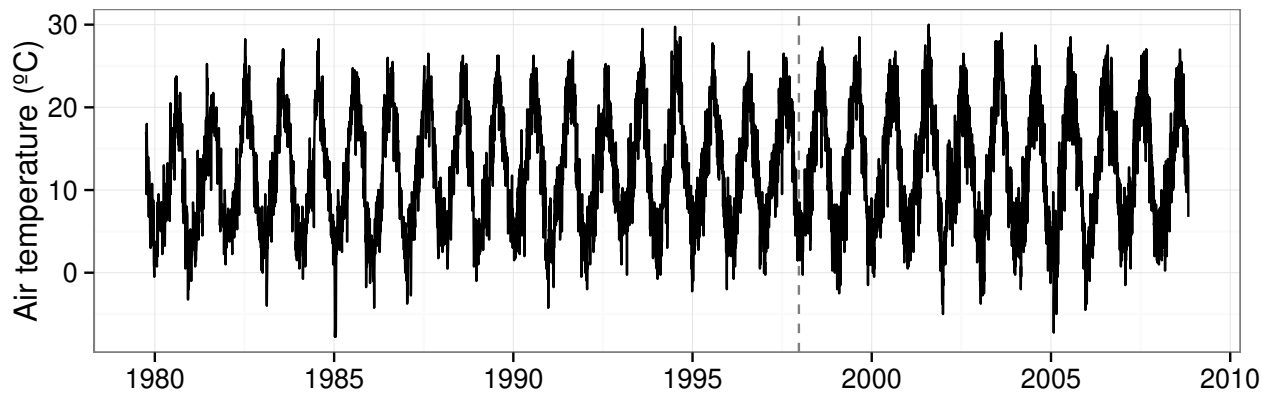

Figure 3. Time series of the mean air temperature at La Baells dam site. 


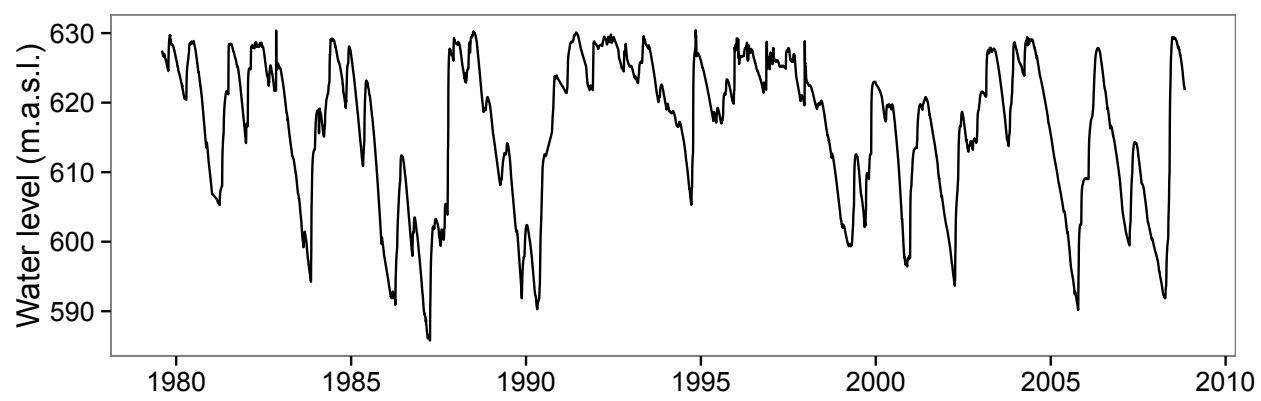

Figure 4. Time series of the reservoir level at La Baells Dam.

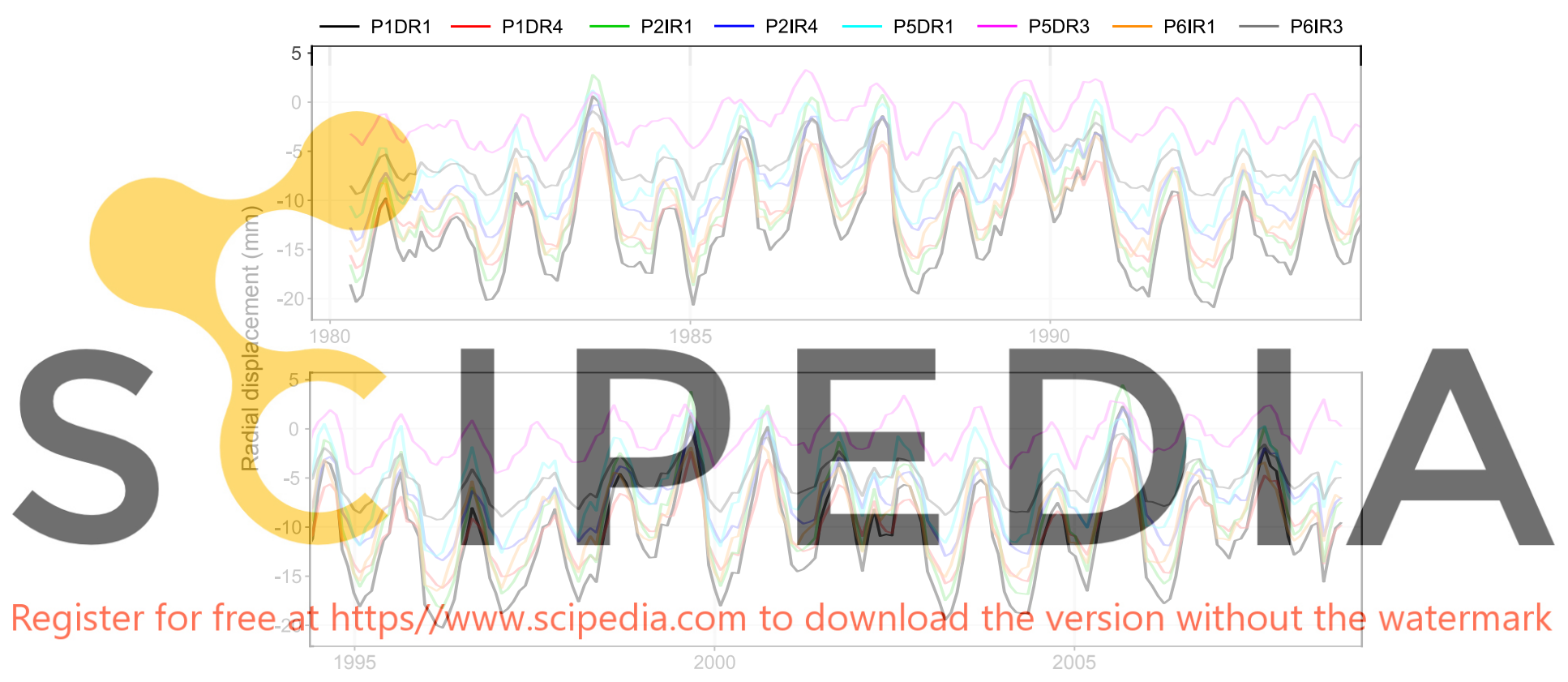

Figure 5. Time series of radial displacements at La Baells Dam. The location of the devices is depicted in Figure 7.

The dam was considered as a three-dimensional solid discretised in hexahedral serendipity 27node elements. A portion of the foundation was also included, resulting in a total of 13,029 nodes and 2,530 elements. The thermal and mechanical problems were solved separately on the resulting finite element mesh (Figure 6), generated with the software GiD [35]. The material properties are shown in table II.

Table II. Material properties considered in the FE model

\begin{tabular}{lll}
\hline Property & Dam & Foundation \\
\hline Young modulus $\left(N \cdot \mathrm{m}^{-2}\right)$ & $4.76 \cdot 10^{10}$ & $3.10 \cdot 10^{10}$ \\
Poisson ratio & 0.25 & 0.25 \\
Density $\left(\mathrm{kg} \cdot \mathrm{m}^{-3}\right)$ & 2,400 & 3,000 \\
Thermal conductivity $\left(\mathrm{W} \cdot{ }^{\circ} \mathrm{K}^{-1} \cdot \mathrm{m}^{-1}\right)$ & 2.4 & 2.2 \\
Thermal expansion coeficient & $10^{-5}$ & $10^{-5}$ \\
Specific heat $\left(\mathrm{J} \cdot \mathrm{kg}^{-1} \cdot{ }^{\circ} \mathrm{K}^{-1}\right)$ & 982 & 950 \\
\hline
\end{tabular}




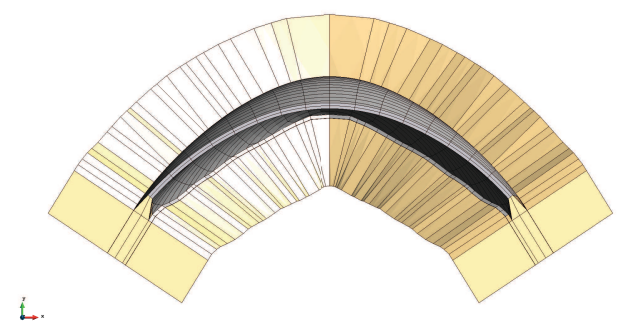

(a) Plan view

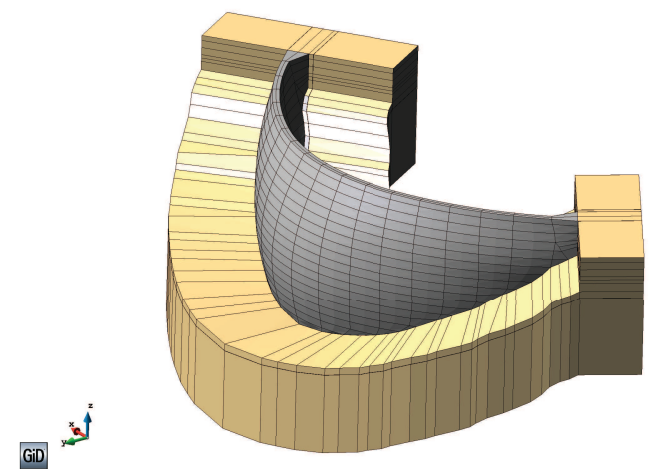

(b) Perspective from upstream

Figure 6. FE model.
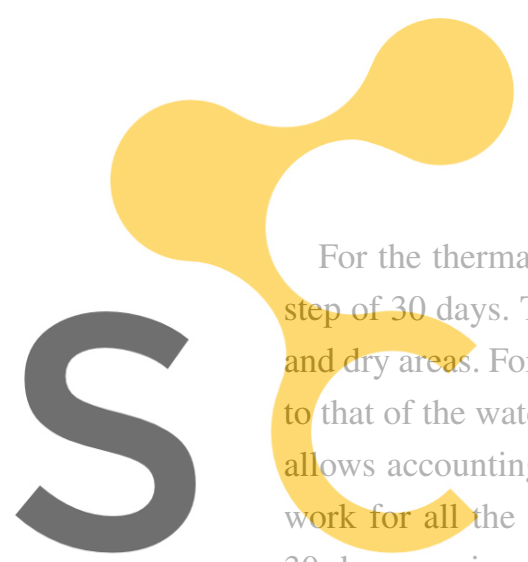

For the thermal problem, a transient computation was run over the 1981-2008 period with time step of 30 days. The temperature was imposed in both dam faces, with different values for the wet and dry areas. For the boundaries belom the reservoir level, the temperature was considered as equal to that of the water, which in turn was estimated allows accounting for work for all the wetted boundaries, equal to tha

30-days moving average of air temperature was imposed, to take into account the thermal inertia.

Register for free result was increased by 2 degrees to account for the solar radiation, following the apprqach proposed by Perez and Martinez for Spanish dams in the North-East region [37]. The temperature

evolution for the first year was repeated 4 times to ensure that the result was not influenced by the initial conditions.

The mechanical response was assumed to be elastic and instantaneous (without inertia), hence for each time step, the hydrostatic load correspondent to the actual reservoir level was applied.

The results of both models (thermal and mechanical) were added, and the displacement evolution at the location of the monitoring devices was extracted. The model results, which are generated in global axes, were later transformed to the local axes correspondent to the radial displacements, as measured by the monitoring devices.

Finally, weekly values were obtained via interpolation, according to the average reading frequency for the available data.

In addition to radial displacements, also the temperature evolution in the dam body was compared to observed data from several thermometers embedded in the dam body.

The goodness of fit of the FE model was computed in terms of the mean absolute error (MAE):

$$
M A E=\frac{\sum_{i=1}^{N}\left|y_{i}-F e m\left(x_{i}\right)\right|}{N}
$$

where $N$ is the number of observations, $y_{i}$ are the observed values and $\operatorname{Fem}\left(x_{i}\right)$ the FEM model results. 


\subsection{Anomalies}

As described in the previous section, the reference time series were those obtained with the FEM model for the 1980-2008 period, where the boundary conditions and loads correspond to the reservoir level and air temperature actually measured in the dam site. Three different types of anomalies were later introduced to modify those data:

- Scenario 1: Progressive breakdown of an isolated device. An increasing value was added to the reference series, with constant rate $\left(a \mathrm{~mm} \cdot\right.$ year $\left.^{-1}\right)$.

- Scenario 2: The same as scenario 1, though the magnitude of the deviation is constant $(a \mathrm{~mm})$

- Scenario 3: Imposed displacement of the left abutment. The data for this scenario were obtained from a modified FEM model representing a hypothetical sliding of the left abutment. For that purpose, the boundary condition at that region was set to $a \mathrm{~mm}$ both in $x$ and $y$ axes (instead of null displacement, as for the reference case).

It is important to note that the anomaly of scenario 3 affects differently to each of the devices analysed. Since a displacement in the left abutment was imposed, the results in the left half of the dam body are anomalous. However, those in the right half are not affected. This can be observed in Figure 7, which depicts the displacement field in the dam body generated by the imposed anomaly with $a=2 m m$.

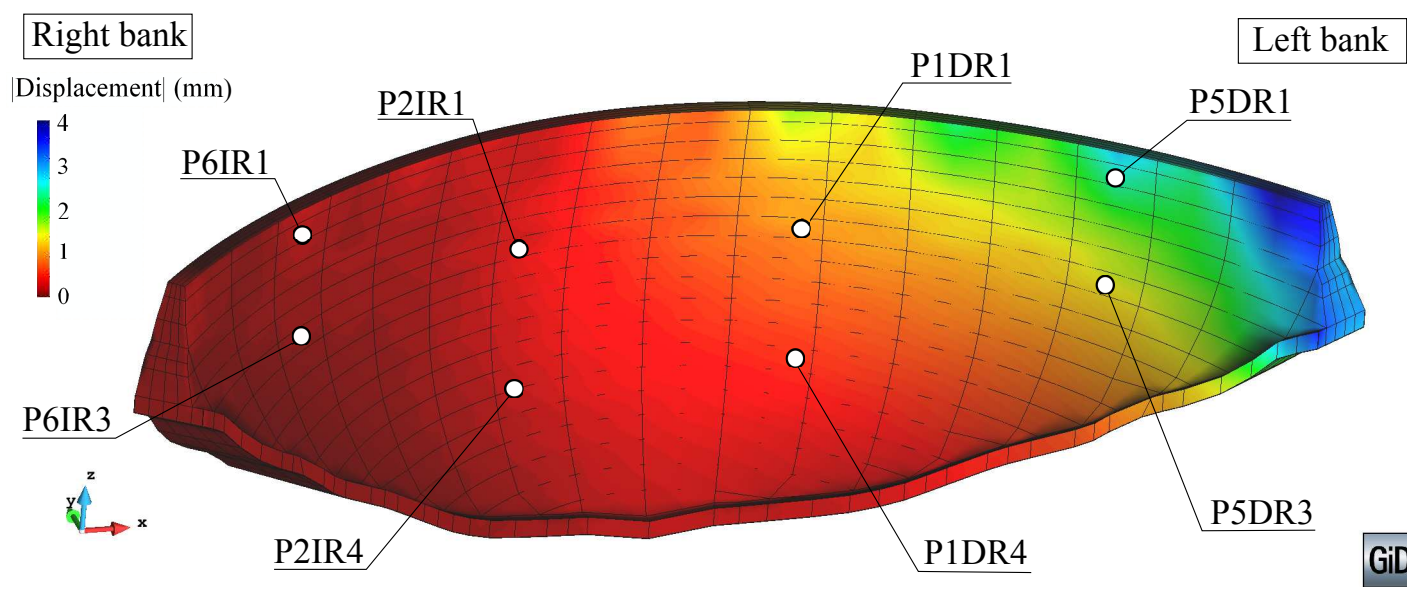

Figure 7. Displacement field resulting from the anomaly in scenario 3. View from downstream.

Table III contains the mean absolute deviation between the reference and the anomalous time series for each device for $a=2 \mathrm{~mm}$. Since the anomaly in scenario 3 does not affect to some devices, those values considered as abnormal by the system will be false positives.

For each scenario, the performance of the three models considered (causal, Non-Causal and autoregressive) was analysed. 4,000 anomalous cases were generated, where the following parameters were randomly selected:

- Initial date of abnormal period

- Anomaly scenario

- Output variable

- Magnitude: $0.5,1.0$ or $2.0 \mathrm{~mm} \cdot$ year $^{-1}$ for scenario $1 ; 0.5,1.0$ or $2.0 \mathrm{~mm}$ for scenario $2 ; 1.0$ or $2.0 \mathrm{~mm}$ for scenario 3 . 
Table III. Discrepancy between the normal displacements, as computed with the FEM model, and those imposed in scenario 3 for $a=2 \mathrm{~mm}$. Mean absolute error ( $\mathrm{mm})$

\begin{tabular}{llll}
\hline Device & MAE $(\mathrm{mm})$ & Device & MAE $(\mathrm{mm})$ \\
\hline P1DR1 & 0.61 & P5DR1 & 1.42 \\
P1DR4 & 0.52 & P5DR3 & 1.05 \\
P2IR1 & 0.10 & P6IR1 & 0.02 \\
P2IR4 & 0.13 & P6IR3 & 0.01 \\
\hline
\end{tabular}

Each anomalous case was presented to all three models to compare their ability for anomaly detection. This was computed in terms of the detection time $\left(t_{d e t}\right)$, defined as the elapsed time from the start of the anomaly until the first observation considered anomalous by each model, measured in days (Figure 8). Since the abnormal period was limited to 1 year, the models which did not detect any anomaly were assigned a $t_{\text {det }}$ value of 365 days.

Moreover, the effectiveness of an anomaly detection system also depends on the number of false positives (observations considered abnormal by the model, which are actually normal) and false negatives (abnormal values not detected as such by the model). The two most commonly used metrics to account for these are precision (2) and recall (3). In this paper, the comparison was mainly based on the $F_{2}$ index (4) [14], which jointly considers precision and recall, giving more importance to the latter.

$$
\begin{gathered}
\text { precision }=\frac{\text { true positives }}{\text { true positives }+ \text { false positives }} \\
\text { recall }=\frac{\text { true positives }}{\text { true positives }+ \text { false negatives }} \\
F_{2}=\left(1+2^{2}\right) \frac{\text { precision } \cdot \text { recall }}{4 \cdot \text { precision }+ \text { recall }}
\end{gathered}
$$

However, these indexes are not useful for model performance assessment when analysing the unaffected devices in scenario 3. In these cases, there are not true positives (all records are normal, since these devices are not affected by the anomaly). Hence, both precision and recall equal zero. Nonetheless, it is highly relevant to know whether the proposed models correctly identify these records within the prediction interval. For that purpose, scenario 3 was analysed by means of the amount of false positives, whose computation depends on the device. For those in the left half of the dam body (as viewed from upstream), which are actually anomalous, the observations above the upper limit of the prediction interval are considered as false positives, since they would imply a deviation towards upstream (while the actual anomaly corresponds to a displacement in the downstream direction). By contrast, for the unaffected devices, every record outside the prediction interval is a false positive, both above the upper limit and below the lower limit of the interval.

\subsection{Load combination verification}

In general, model accuracy is dependent on the values of the input variables. The more input data available for similar situations to that to be predicted, the more accuracy is to be expected. In dam behaviour, it will depend on the thermal and hydrostatic loads. 
This effect is more important when input values are out of the training data range [38]. In particular, the accuracy of data-based models as BRTs may decrease dramatically when extrapolating.

Cheng et al. [15] defined a possible abnormal state of the dam (State 3), that "may be caused by extreme environmental values variables". In this work, this issue was explicitly verified, and out-of-range (OOR) instances were considered as potential false positives.

This verification was carried out following an original procedure, specifically designed for the dam behaviour problem, where there are three main loads: thermal, mechanical (hydrostatic head) and temporal.

If the behaviour of the dam does not change over time, the importance of time variable is negligible. This was checked when fitting BRT models to the reference data, which correspond to time-independent dam behaviour. The inclusion of these variables is useful for retrospective analysis, as confirmed by previous studies [11]. In practice, a previously trained model is employed to predict future values. Hence, it is obvious that the model prediction is an extrapolation in time axis and thus does not need to be verified.

As for the other two loads (thermal and hydrostatic), the simplest approach would be to check whether their values for the test period are greater (lower) than the maximum (minimum) within the training data set. However, that would not consider that both effects are coupled: the water temperature is different to that of the air, hence the water surface elevation affects the boundary condition in the upstream dam face and, as a result, conditions the thermal response of the dam [39].

Moreover, there is not a widely accepted agreement on what extrapolation is and how to handle it [38]. In dam behaviour modelling, it seems obvious that a hydrostatic load above the maximum in the training set is out-of-range. However, a more detailed definition seems appropriate to account for the "empty space phenomenon" [40], i.e., the existence of areas without training samples within the range of the inputs.

To account for this issue, the criterion employed in this work is based on the combination of both loads:

1. The training data are plotted in the (Reservoir level, Air temperature) plane.

2. A two-dimensional density function is computed by means of the kernel density estimation (KDE) method.

3. The training instance with lower density value is localised, and the corresponding isoline is plotted.

4. The input values for the new data are plotted on the same plane. Those falling outside the isoline are considered as out-of-range.

With this procedure, it is taken into account that the predictive accuracy can be poor for a load combination not previously presented, even though their values, if considered separately, are within the training range. An example of this issue is presented in Figure 8. 

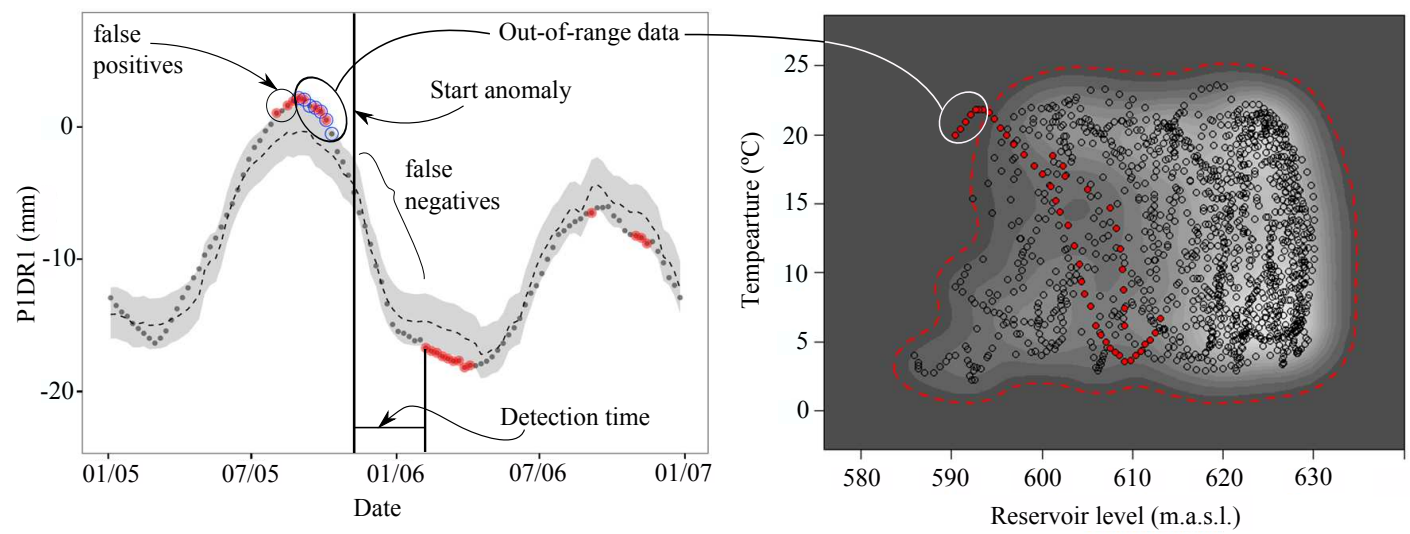

Figure 8. Model performance indicators. Left: typical output plot, with the observations (circles), the predictions (dotted line), and the prediction interval (shaded area). Before the start of anomaly, some data fall outside the prediction interval (in red). Of those, some are false positives, whereas others correspond to out-of-range inputs (blue circles), since they fall in a low-density region in the 2D density plot (right). In this case, a combination of high temperature and low reservoir level was presented for the first time in dam history.

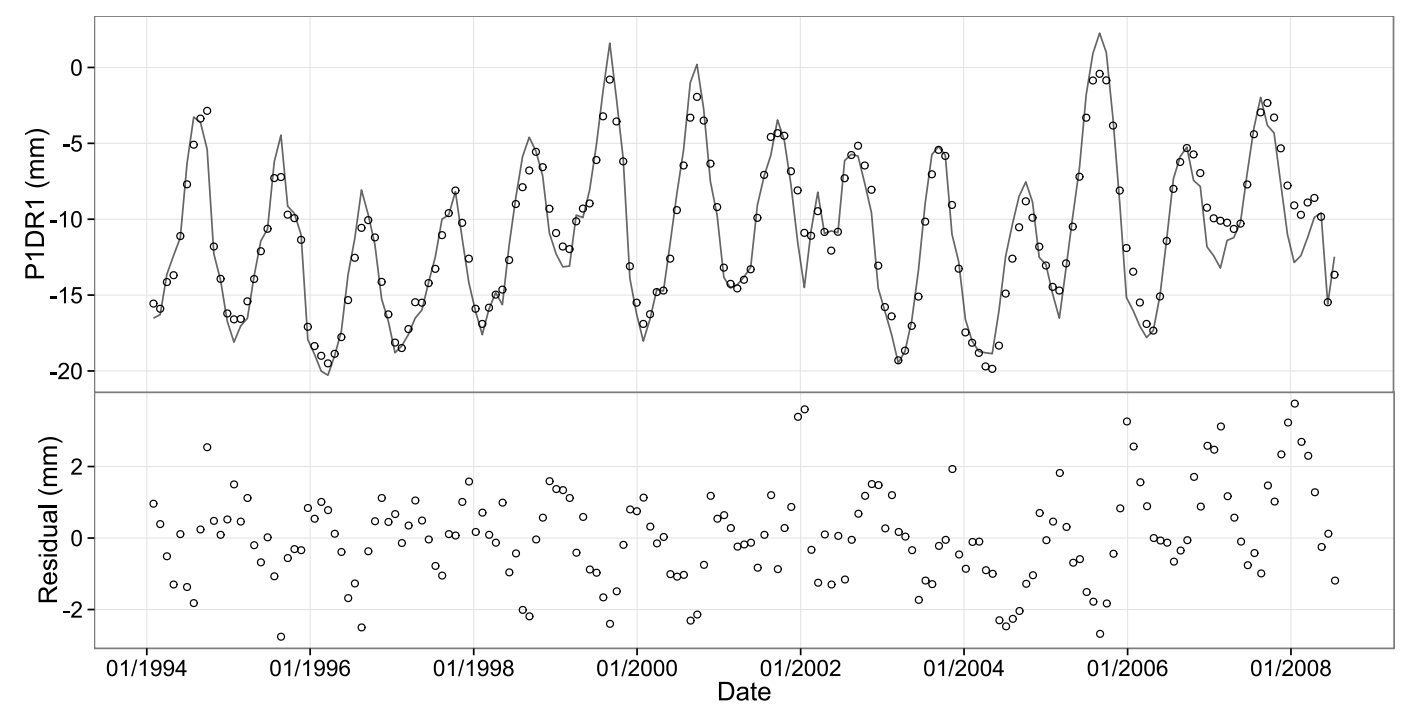

Figure 9. FEM results versus observations for P1DR1

\section{RESULTS AND DISCUSSION}

\subsection{FE model accuracy}

Figure 9 shows the comparison between the observed radial displacements for P1DR1 and those obtained with the FE model for the period 1994-2008. Results for other outputs are similar (Table IV). The FEM model accuracy is comparable to that obtained in previous studies with data-based models [8].

As regards the temperature, Figure 10 shows the numerical results and the observed data for 4 thermometers and the January 2007 - June 2008 period. Both the devices and the time period 
Table IV. Deviation between the radial displacements as computed with the FEM and the actual records for the 1994-2008 period. Mean absolute error

\begin{tabular}{llll}
\hline Output & MAE $(\mathrm{mm})$ & Output & MAE $(\mathrm{mm})$ \\
\hline P1DR1 & 0.70 & P5DR1 & 0.81 \\
P1DR4 & 0.65 & P5DR3 & 1.01 \\
P2IR1 & 1.08 & P6IR1 & 0.96 \\
P2IR4 & 0.98 & P6IR3 & 0.58 \\
\hline
\end{tabular}
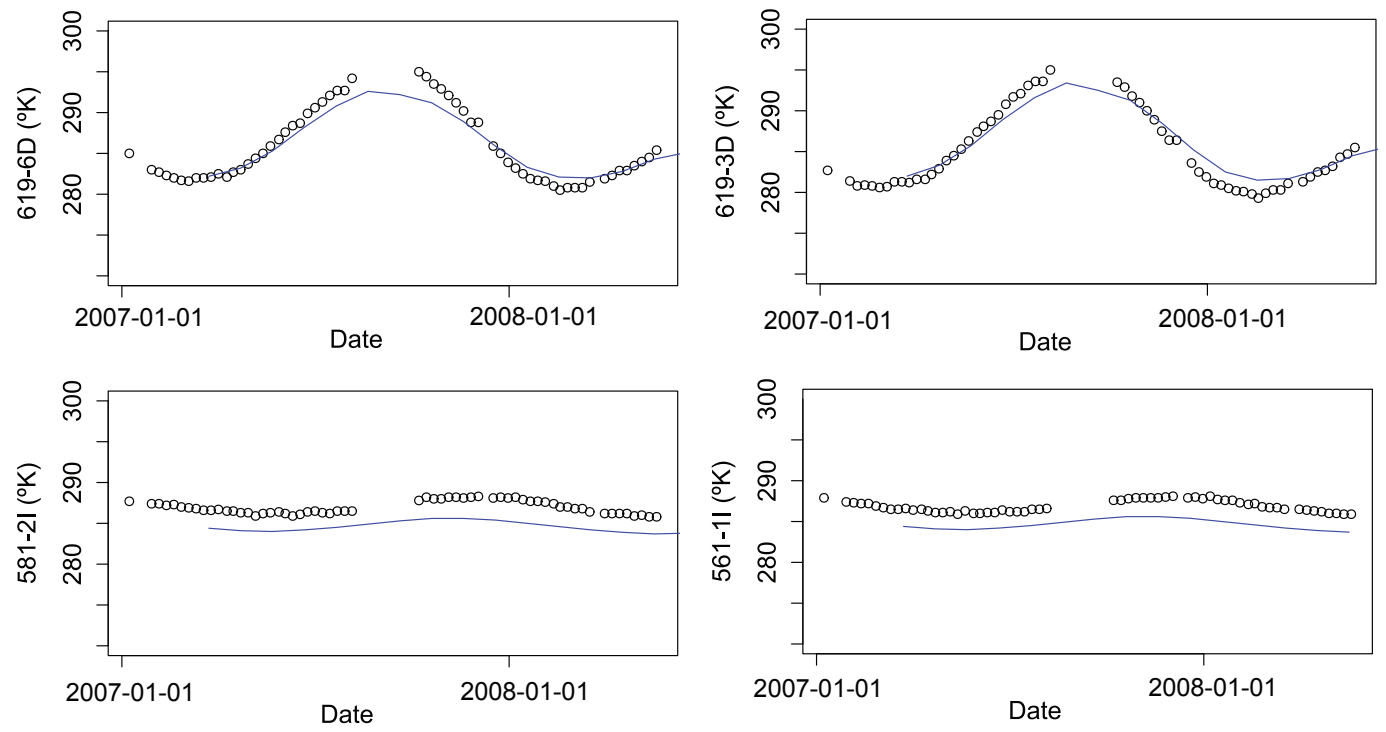

Figure 10. Comparison between numerical and measured temperature in 4 locations within the dam body

correspond to the results published by Santillán et al. [41], who employed a highly detailed thermal model, also for La Baells Dam.

Since this study does not specifically focus on predicting the thermal response, relevant simplifications were employed to generate the reference data (neglecting the variation in water temperature with depth, using a relatively large time step). Nonetheless, the temperature within the dam body was well captured.

This, together with the results for displacements, confirm that the resulting data series mostly reproduce the dam response to the main loads. Therefore, they are representative of the normal behaviour of the dam and useful to evaluate the ability of the methodology to detect anomalies.

\subsection{Prediction accuracy}

The performance of all models on the reference data (without anomalies) was first assessed. The objectives are a) verify the evolution of the prediction accuracy over time (as more data is included in the training set), b) check the effect of averaging the standard deviation, c) compare all models in terms of false positives, and d) evaluate the efficiency of the criterion to detect out-of-range data.

For that purpose, the iterative process described in section 2.2 was followed, i.e., each model was re-fitted yearly over an increasing training set, and the prediction interval was updated as a function 

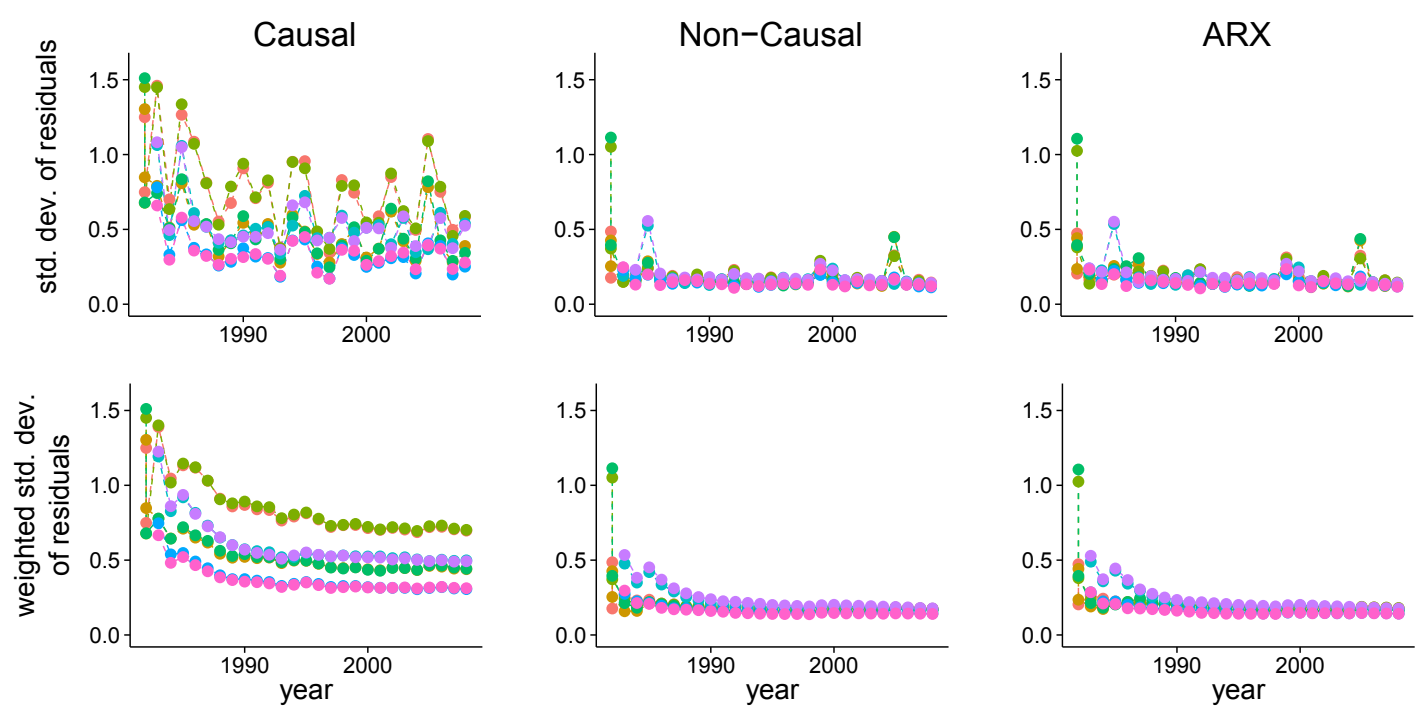

- P1DR1 • P1DR4 • P2IR1 - P2IR4 • P5DR1 • P5DR3 • P6IR1 • P6IR3

Figure 11. Time evolution of the prediction accuracy for all models and outputs. Top: standard deviation of residuals per year. Bottom: weighted average.

of the actualised value of the weighted average of the residual standard deviation. Since the damfoundation behaviour is time-independent for the reference case, the variation in model accuracy is due to the increase of training data.

Figure 11 shows the evolution of both the raw and the weighted average of the residual standard deviation for all devices and models. Some conclusions can be drawn:

- As expected, the accuracy of the Non-Causal and ARX models model is higher, since the non-causal inputs implicitly contain information regarding external variables not considered in the causal version.

- The inclusion of lagged variables in the ARX model is not relevant, as compared to the NonCausal one.

- The raw values show high variance, especially for the causal model, which is eliminated by averaging

- The time evolution of the weighted standard deviation of the residuals is similar for all models: a sharp decrease in the first years, followed by quasi-constant behaviour. Nonetheless, the causal model requires more data to reach the low-slope part of the curve.

Table V contains the amount of false positives for all targets and models, as well as those correspondent to out-of-range inputs. Although the prediction interval for the causal model is wider (due to the higher residual standard deviation), it also generates a greater quantity of false positives. However, the average amount is low in all cases, as compared to the total amount of records $(1,464)$. Moreover, the procedure to identify out-of-range inputs reduces the false positives by $27 \%$ for the causal model and by $45 \%$ for both the Non-Causal and the ARX. As a result, the mean percentage of false positives is 8.0, 2.8 and $2.6 \%$ respectively. It should be noticed that the results for the NonCausal and ARX models are lower than the theoretical percentage of values outside the interval within 2 times the standard deviation in a normal distribution (5\%). 
Table V. Amount of false positives

\begin{tabular}{lllllll}
\hline Model & \multicolumn{2}{c}{ Causal } & \multicolumn{2}{c}{ Non-Causal } & \multicolumn{2}{c}{ ARX } \\
\hline Target & \# False pos. & \# OOR & \# False pos. & \# OOR & \# False pos. & \# OOR \\
\hline P1DR1 & 179 & 53 & 91 & 40 & 82 & 35 \\
P1DR4 & 178 & 54 & 89 & 42 & 75 & 38 \\
P2IR1 & 184 & 54 & 89 & 41 & 85 & 35 \\
P2IR4 & 198 & 54 & 95 & 50 & 75 & 38 \\
P5DR1 & 125 & 31 & 50 & 21 & 51 & 21 \\
P5DR3 & 164 & 49 & 72 & 31 & 68 & 30 \\
P6IR1 & 129 & 31 & 51 & 21 & 50 & 21 \\
P6IR3 & 171 & 42 & 63 & 27 & 65 & 28 \\
\hline Mean & 166 & 46 & 75 & 34 & 69 & 31 \\
\hline
\end{tabular}

\subsection{Anomaly detection}

Figure 12 (a) shows the $F_{2}$ results as a function of the model and the anomaly magnitude $a$ for scenarios 1 and 2. As expected, the larger anomalies were more easily detected in all cases. As for the input variables, Non-Causal model performed better on average, especially for small anomalies and as compared to the causal model. Again, the inclusion of lagged variables generated a minor effect, in this case towards slightly poorer performance.
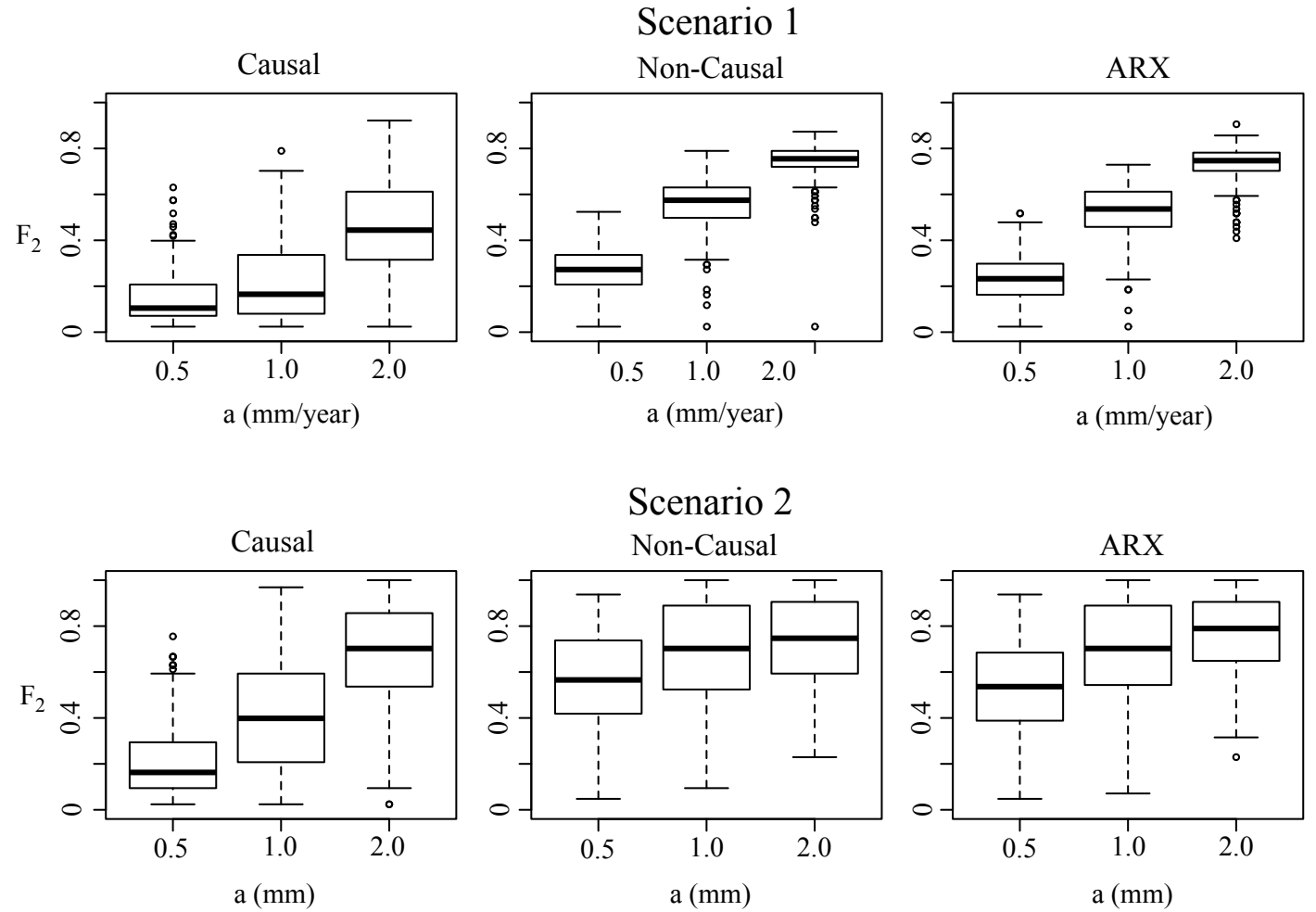

Figure 12. $F_{2}$ index for scenarios 1 and 2.

The results for Scenario 3 are more interesting to analyse, since they correspond to a realistic anomaly affecting the overall dam behaviour. Since the effect of this anomaly is different to each 

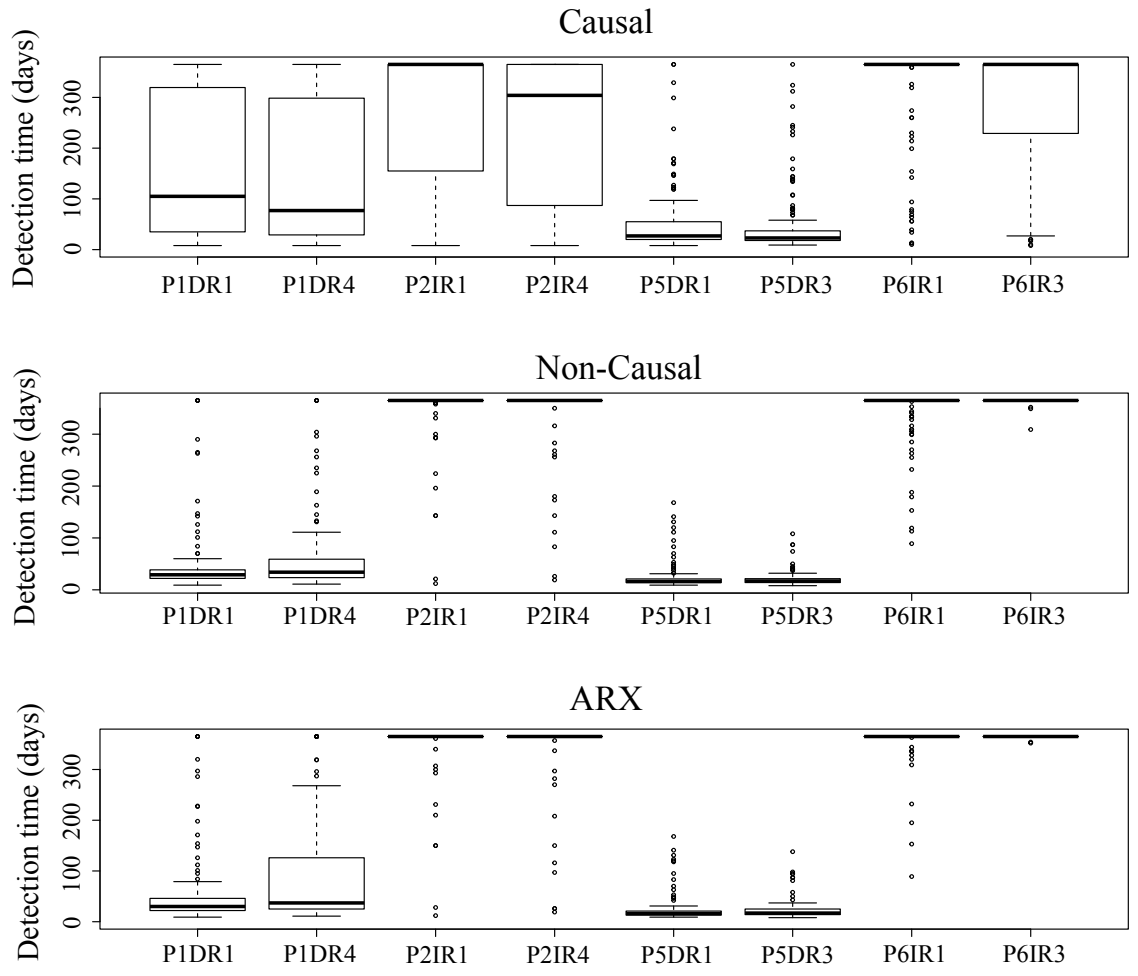

Figure 13. Detection time (days) per target and model for scenario 3.

output, the results are presented in terms of the true detection time $t_{d}$ per device, i. e. the elapsed time until the first record identified as a deviation towards downstream. Figure 13 shows the results.

A perfect model would feature null detection time for the affected devices (P1DR1, P1DR4, P5DR1 and P5DR3), and 365 days for the remaining (P2IR1, P2IR4, P6IR1 and P6IR3). Both the Non-Causal and the ARX models showed almost perfect performance. As regards the causal model, the anomaly in the most affected devices (P5DR1 and P5DR3) is detected almost instantly, but is less effective for P1DR1 and P1DR4, whose deviation from the reference behaviour is low (see Table IV). The detection time for P1DR1 and P1DR4 is around two months, with high variation up to 300 days.

A complete assessment of the model performance requires analysing the amount of false positives. They correspond to any value outside the prediction interval for the targets in the right half of the dam body, and to anomalies correspondent to deviations towards upstream for those in the left region. Figure 14 shows these results.

It can be observed that the causal model is clearly more effective in this regard: both the NonCausal and the ARX models classify around half of the observations for the unaffected devices as abnormal (there are 52 observations in the period of analysis). This result is due to the nature of the inputs for each model. For example, the Non-Causal model generates a prediction for P6IR1 based on the value of P5DR1 (among other inputs, but this is particularly important for being symmetrical within the dam body). In scenario 3, P5DR1 deviates towards downstream with respect to the reference (training) period. Since that input is anomalous, the resulting prediction is also wrong. In this case, the model interprets that the value of P6IR1 falls in the upstream side of the prediction interval. 

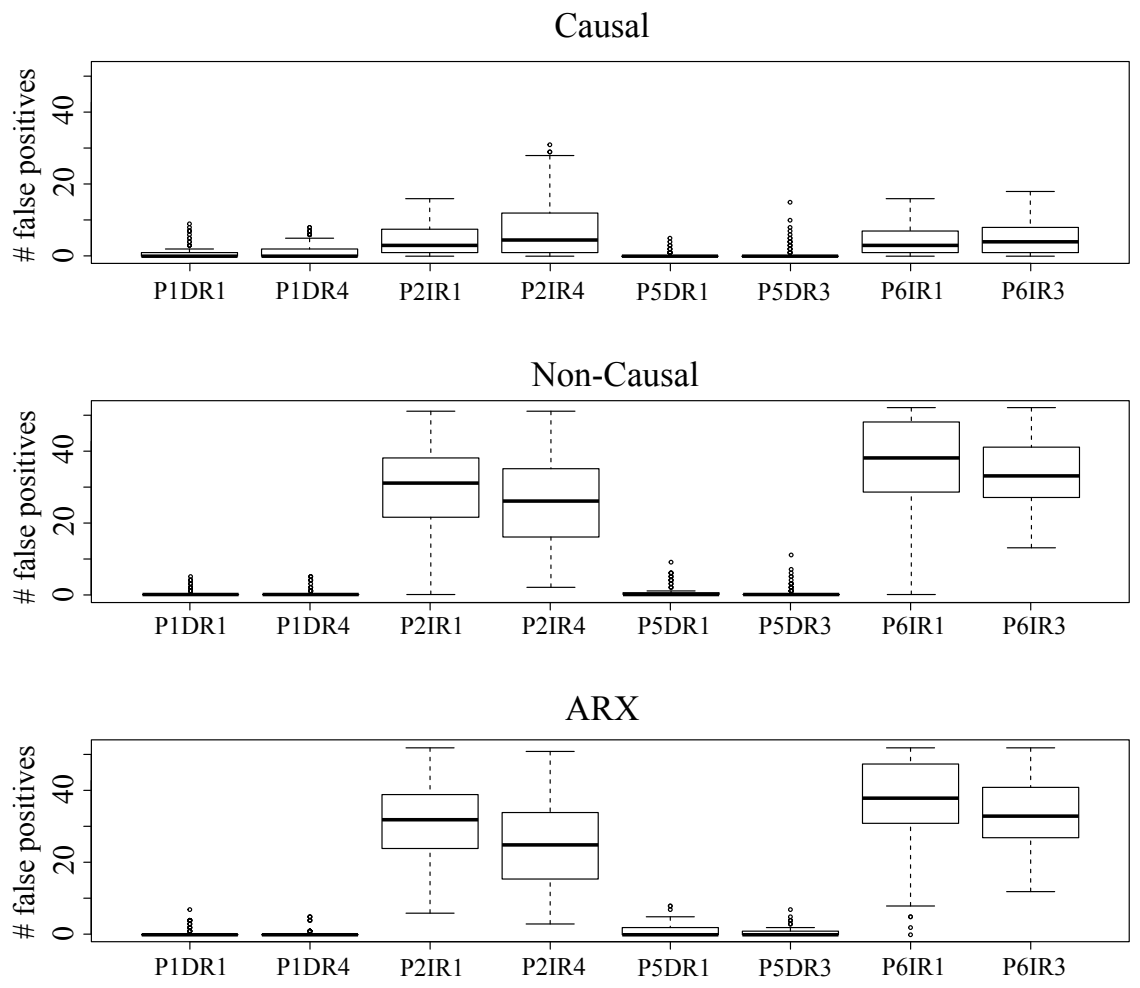

Figure 14. False positives per target and model for scenario 3.

This issue is highly relevant, since the final aim of the system is not only to detect a potentially anomalous behaviour, but also to support the correct identification of the cause, and then the decision making. In fact, similar results would have been obtained had the devices been analysed jointly in scenarios 1 and 2: a real deviation towards downstream in some device is (in general) correctly identified by the non-causal models, but that same value would generate an incorrect prediction for other devices, of opposite sign.

Causal models do not give these spurious results, since they predict the dam response only based on the external variables, at the cost of a generally higher detection time.

A straightforward option to avoid this behaviour is to discard non-causal models. However, their good performance for detecting true anomalies suggests that they can be useful overall.

As an alternative, the outputs whose value is identified as anomalous by a non-causal model can be removed from the input set. The model requires re-training, but it can still offer accurate results, thanks to the flexibility of BRTs.

A new set of 240 cases was run for scenario 3 and the Non-Causal model. The results shown in Figure 15 confirm that the removal of abnormal variables is effective against false positives, while maintaining the ability for anomaly detection. The model performance is only poorer for P2IR1 (unaffected by the anomaly in scenario 3): the detection time is lower than 365 days, which indicates the existence of false positives. Nonetheless, the average detection time is still 270 days, and the total amount of false positives is lower than $10 \%$.

This approach was implemented in a new visualisation tool, which was developed to present the results for all devices involved. It is based on the Shiny library [42], and includes two plots for 

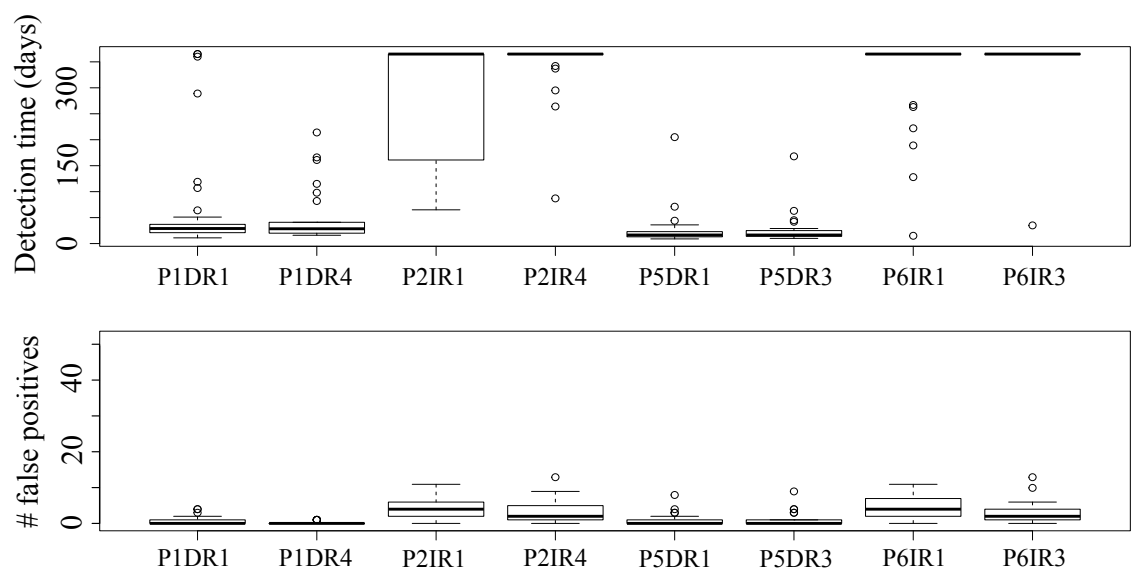

Figure 15. Detection time and false positives per target for scenario 3 and the Non-Causal model, once the anomalous variables are removed from the input set.

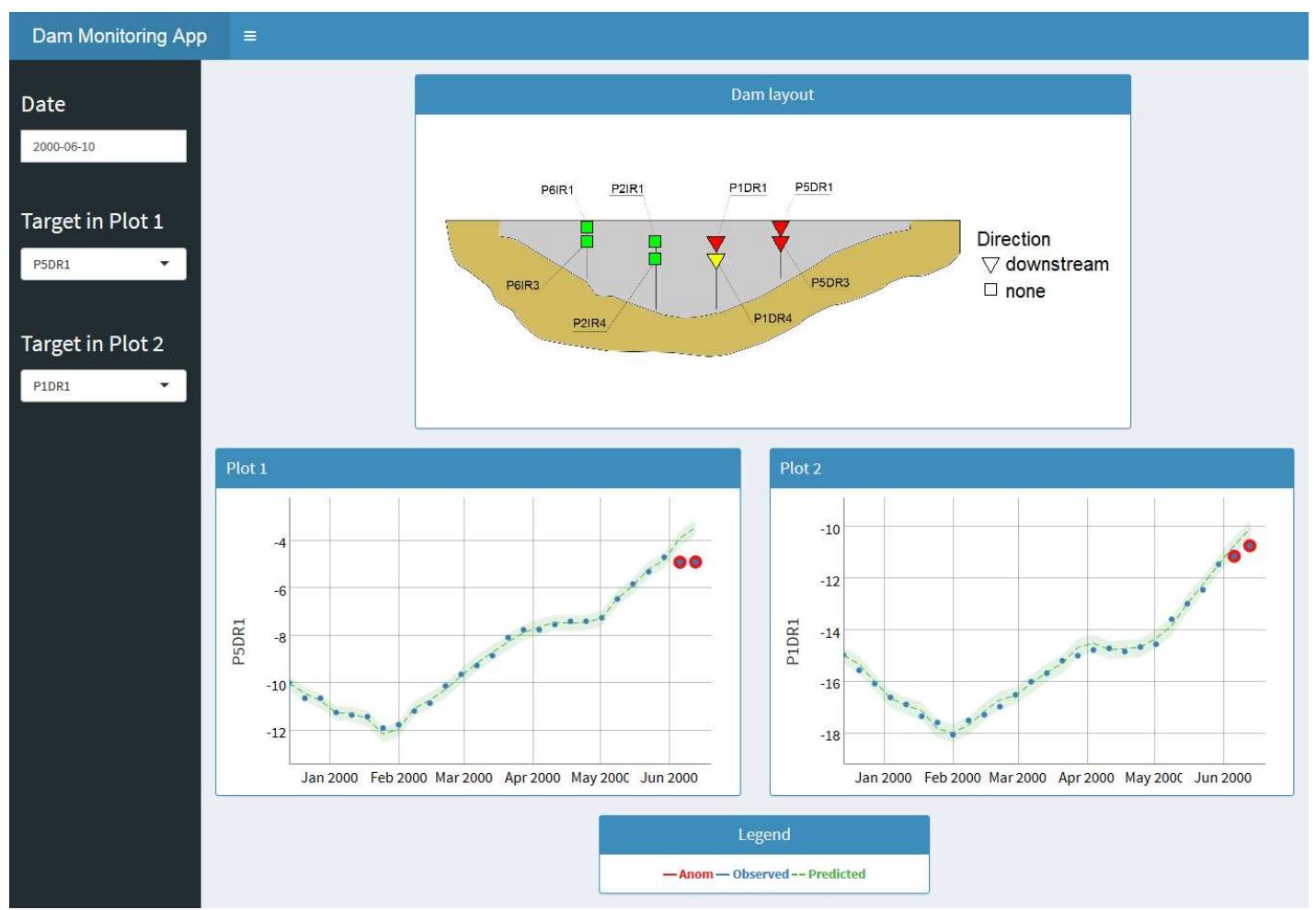

Figure 16. Interface of the dam monitoring data analysis tool for a case from scenario 3 . The imposed displacement in the left abutment is correctly identified

each model (Figure 16). First, each device is plotted on its actual location within the dam body, with a symbol that is a function of the deviation between prediction and observation for the date under consideration. Then, the evolution of observations and predictions for the most recent period is plotted for one device selected by the user. Figure 16 shows the application interface for one of the anomalies from scenario 3. It can be observed that the anomaly is correctly localised.

With this tool, the user jointly receives the overall information on all devices under consideration, and a more detailed plot of the selected output, where the value of the deviation, as well as the trend, 
can be observed. In this version, devices whose residuals are lower than two times the standard deviation are plotted in green; those between two and three times are depicted in yellow, and those above three times are shown in red. The shapes correspond to the direction of the deviation (upstream or downstream), as interpreted by each model. This criterion can be tailored to the user preferences.

\section{SUMMARY AND CONCLUSIONS}

A methodology for early detection of anomalies in dam behaviour was presented, which includes a prediction model based on BRT, a criterion for detecting anomalies based on the residual density function, and a procedure for realistic estimation of the prediction interval. Also, extraordinary loads are identified by jointly considering the two most important external loads (hydrostatic load and temperature).

Causal models (which only consider external variables) and non-causal (including both internal and lagged variables as predictors) were compared in terms of detection time for three different anomaly scenarios. The results showed that non-causal models are more effective for the detection of anomalies, both affecting to isolated devices (Scenarios 1 and 2), and those resulting from an overall malfunction of the dam (Scenario 3).

In the case study considered, the inclusion of lagged variables had minor effect both in the model accuracy and the detection time. This suggests that the Non-Causal model (without lagged variables) might be a better choice due to its higher simplicity.

Causal models were more robust as regards the precision (when accounting for false positives). In abnormal periods, the prediction of non-causal models for unaffected devices is often wrong because it is partially based on anomalous data (that from the devices actually affected by the anomaly). This type of behaviour is a consequence of the nature of the model itself, and is the price to pay in exchange for a greater ability for early detection of anomalies.

However, an updated version of the Non-Causal model, where the anomalous variables are removed from the input set, avoided the above-mentioned issue, and showed to be as effective for anomaly detection as the Non-Causal, and even more robust against false positives than the causal model. Hence, this approach is the best option to provide useful information to the dam safety managers. To that end, it was implemented in an interactive on-line tool, which shows the devices whose behaviour is interpreted as potentially abnormal by the predictive model, together with the plot of the evolution of predictions and observations for all relevant outputs.

This tool can be used as a support for decision making, since it facilitates the identification of a potential deviation from normal behaviour. Thus, it can be used as an indicator to generate a warning which might lead to intensify the dam safety monitoring activity. Nonetheless, all relevant decisions influencing dam safety should be made by an expert and capable engineer, based on the analysis of all the relevant information available. 


\section{ACKNOWLEDGEMENTS}

The authors thank Carlos Barbero, dam safety manager at the Catalan Water Agency, for providing the monitoring data.

The research was supported by the Spanish Ministry of Economy and Competitiveness (Ministerio de Economía y Competitividad, MINECO) through the projects iComplex (IPT-2012-0813-390000) and AIDA (BIA2013-49018-C2-1-R and BIA2013-49018-C2-2-R).

\section{REFERENCES}

1. International Commission on Large Dams. Dam surveillance guide. Technical Report B-158, ICOLD 2012.

2. Mata J, Tavares de Castro A, Sá da Costa J. Constructing statistical models for arch dam deformation. Structural Control and Health Monitoring 2014; 21(3):423-437.

3. Mata J. Interpretation of concrete dam behaviour with artificial neural network and multiple linear regression models. Engineering Structures 2011; 3(3):903 - 910, doi:10.1016/j.engstruct.2010.12.011.

4. Ranković V, Grujović N, Divac D, Milivojević N. Development of support vector regression identification model for prediction of dam structural behaviour. Structural Safety 2014; 48:33-39.

5. Su H, Chen Z, Wen Z. Performance improvement method of support vector machine-based model monitoring dam safety. Structural Control and Health Monitoring 2016; 23(2):252-266.

6. Ranković V, Grujović N, Divac D, Milivojević N, Novaković A. Modelling of dam behaviour based on neuro-fuzzy identification. Engineering Structures 2012; 35:107-113, doi:10.1016/j.engstruct.2011.11.011.

7. Salazar F, Morán R, Toledo MÁ, Oñate E. Data-based models for the prediction of dam behaviour: A review and some methodological considerations. Archives of Computational Methods in Engineering 2015; :1-21.

8. Salazar F, Toledo M, Oñate E, Morán R. An empirical comparison of machine learning techniques for dam behaviour modelling. Structural Safety 2015; 56:9-17.

9. Myers B, Scofield D. Providing improved dam safety monitoring using existing staff resources: Fern Ridge Dam case study. Proceedings of 28th Annual USSD Conference, 2008.

10. $\mathrm{Yu} \mathrm{H}, \mathrm{Wu} \mathrm{Z}, \mathrm{Bao} \mathrm{T}$, Zhang L. Multivariate analysis in dam monitoring data with PCA. Science China Technological Sciences 2010; 53(4):1088-1097, doi:10.1007/s11431-010-0060-1.

11. Salazar F, Toledo MÁ, Oñate E, Suárez B. Interpretation of dam deformation and leakage with boosted regression trees. Engineering Structures 2016; 119:230-251.

12. Mata J, Leitão NS, de Castro AT, da Costa JS. Construction of decision rules for early detection of a developing concrete arch dam failure scenario. a discriminant approach. Computers \& Structures 2014; 142:45-53.

13. Hodge VJ, Austin J. A survey of outlier detection methodologies. Artificial Intelligence Review 2004; 22(2):85-126.

14. Jung IS, Berges M, Garrett JH, Poczos B. Exploration and evaluation of ar, mpca and kl anomaly detection techniques to embankment dam piezometer data. Advanced Engineering Informatics 2015; 29(4):902-917.

15. Cheng L, Zheng D. Two online dam safety monitoring models based on the process of extracting environmental effect. Advances in Engineering Software 2013; 57:48-56.

16. Gamse S, Oberguggenberger M. Assessment of long-term coordinate time series using hydrostatic-season-time model for rock-fill embankment dam. Structural Control and Health Monitoring 2016; .

17. Kao CY, Loh CH. Monitoring of long-term static deformation data of Fei-Tsui arch dam using artificial neural network-based approaches. Structural Control and Health Monitoring 2013; 20(3):282-303.

18. Li F, Wang Z, Liu G. Towards an error correction model for dam monitoring data analysis based on cointegration theory. Structural Safety 2013; 43:12-20.

19. Loh $\mathrm{CH}$, Chen $\mathrm{CH}$, Hsu TY. Application of advanced statistical methods for extracting long-term trends in static monitoring data from an arch dam. Structural Health Monitoring 2011; 10(6):587-601.

20. Breiman L, Friedman JH, Olshen RA, Stone CJ. Classification and regression trees. Wadsworth \& Brooks: Monterrey, CA, 1984.

21. Friedman J. Greedy function approximation: a gradient boosting machine. Annals of Statistics 2001; :1189 - 1232.

22. Ridgeway G. Generalized Boosted Models: A guide to the gbm package 2007. URL http://CRAN. $\mathrm{R}$-project. org/package=gbm, r package vignette.

23. Leathwick J, Elith J, Francis M, Hastie T, Taylor P. Variation in demersal fish species richness in the oceans surrounding new zealand: an analysis using boosted regression trees. Marine Ecology Progress Series 2006; 
321:267-281.

24. Elith J, Leathwick JR, Hastie T. A working guide to boosted regression trees. Journal of Animal Ecology 2008; 77(4):802-813.

25. Schapire RE. The boosting approach to machine learning: An overview. Nonlinear estimation and classification. Springer, 2003; 149-171.

26. Michelis A. Traditional versus non-traditional boosting algorithms. Master's Thesis, University of Manchester 2012.

27. R Core Team. R: A Language and Environment for Statistical Computing. R Foundation for Statistical Computing, Vienna, Austria 2013. URL http://www. R-project.org/.

28. Ridgeway G. gbm: Generalized Boosted Regression Models 2013. R package version 2.1.

29. Salazar F, González J, Toledo M, Oñate E. A methodology for dam safety evaluation and anomaly detection based on boosted regression trees. Proceedings of the 8th European Workshop on Structural Health Monitoring, Bilbao, Spain, 2016.

30. Hyndman RJ, Athanasopoulos G. Forecasting: principles and practice. OTexts, 2014.

31. Arlot S, Celisse A, et al. A survey of cross-validation procedures for model selection. Statistics surveys 2010; 4:40-79.

32. Herrera M, Torgo L, Izquierdo J, Pérez-García R. Predictive models for forecasting hourly urban water demand. Journal of Hydrology 2010; 387(1):141-150.

33. Friedman JH, Meulman JJ. Multiple additive regression trees with application in epidemiology. Statistics in medicine 2003; 22(9):1365-1381.

34. Palumbo P, Piroddi L, Lancini S, Lozza F. NARX modeling of radial crest displacements of the Schlegeis arch dam. Proceedings of the Sixth ICOLD Benchmark Workshop on Numerical Analysis of Dams, Salzburg, Austria, 2001.

35. Ribó R, Pasenau M, Escolano E, Ronda J, González L. GiD reference manual. CIMNE, Barcelona 1998; .

36. Bofang Z. Prediction of water temperature in deep reservoirs. Dam Engineering 1997; 8:13-26.

37. Pérez J, Martínez E. La acción térmica del medio ambiente como solicitación de diseño en proyectos de presas españolas. Rev Obras Públicas 1995; 3349:79-90. [in Spanish].

38. Ebert T, Belz J, Nelles O. Interpolation and extrapolation: Comparison of definitions and survey of algorithms for convex and concave hulls. Computational Intelligence and Data Mining (CIDM), 2014 IEEE Symposium on, IEEE, 2014; 310-314.

39. Tatin M, Briffaut M, Dufour F, Simon A, Fabre JP. Thermal displacements of concrete dams: Accounting for water temperature in statistical models. Engineering Structures 2015; 91:26-39.

40. Verleysen M, et al.. Learning high-dimensional data. Nato Science Series Sub Series III Computer And Systems Sciences 2003; 186:141-162.

41. Santillán D, Salete E, Vicente D, Toledo M. Treatment of solar radiation by spatial and temporal discretization for modeling the thermal response of arch dams. Journal of Engineering Mechanics 2014; 140(11).

42. Chang W, Cheng J, Allaire J, Xie Y, McPherson J. shiny: Web Application Framework for $R$ 2016. URL http: //CRAN.R-project. org/package=shiny, $\mathrm{r}$ package version 0.13.2. 